\title{
PDAC: Um Protocolo de Alocação Dinâmica de Canais para Ambientes Médicos
}

\author{
Bruno Marques Cremonezi ${ }^{1}$, Alex Borges Vieira ${ }^{1}$, Michele Nogueira ${ }^{2}$ \\ ${ }^{1}$ Depto. de Ciência da Computação - Universidade Federal de Juiz de Fora (UFJF) \\ ${ }^{2}$ Depto. de Informática - Universidade Federal do Paraná (UFPR) \\ brunomarques@ice.ufjf.br, alex.borges@ufjf.edu.br, michele@inf.ufpr.br
}

\begin{abstract}
Resumo. Redes corporais permitem o monitoramento contínuo de pacientes em ambientes médico-hospitalares. Porém, a alta densidade de redes nesses ambientes e a escassez de canais de comunicação provocam interferências e consequente baixo desempenho. A correta alocação de canais pode eliminar problemas deste tipo. Entretanto, as soluções existentes para este problema confiam em entidades centralizadoras e não conseguem prover um serviço que obedeça aos requisitos de aplicações médicas. Nesse sentido, apresentamos PDAC, um protocolo na camada de acesso ao meio de comunicação ciente dos requisitos das aplicações médicas. PDAC inspirado em uma solução gulosa do problema de coloração de grafos e promove uma alocação dinâmica de canais em cenários hospitalares através do trabalho colaborativo de múltiplas estações base. O protocolo teve suas propriedades verificadas formalmente e, os resultados de simulações de ambientes realistas mostram que ele aumenta a vazão média da rede em 30\% e reduz a latência de transferência em 40\%, se comparado com os protocolos de alocação de frequência presentes na literatura.
\end{abstract}

\section{Introdução}

Com a recente popularização das redes corporais sem fio, o número de usuários que utilizam esse tipo de rede aumentou consideravelmente. Sua natureza móvel, em conjunto com a comunicação via radio frequência, torna essas redes mais vulneráveis a problemas inerentes a uma comunicação sem fio. É comum, por exemplo, que duas ou mais redes corporais diferentes utilizem simultaneamente um mesmo canal de comunicação (ou canais parcialmente sobrepostos). Isto potencializa interferências na comunicação e acarreta um maior número de retransmissões e perdas de pacote na rede. Desta forma, essas redes apresentam altas latências e baixa transmissão [Fang et al. 2010].

Entretanto, em um ambiente médico-hospitalar é observada uma alta densidade de redes corporais e locais sem fio [Baker and Hoglund 2008, Fang et al. 2010]. Por possuir uma reduzida faixa de frequência para aplicações médicas, o número de canais sem sobreposição é baixo, tornando o processo de alocação de canais para as muitas redes corporais um problema desafiador. O processo de alocação deve ser rápido para atender aos fortes requisitos das aplicações médicas, em que a latência e entrega de pacotes máxima permitida deve ser, respectivamente, de 200 ms [Baker and Hoglund 2008] e 99\% [Yu et al. 2006].

Para evitar interferências, diversos trabalhos propuseram técnicas de alocação de canais entre redes corporais co-localizadas [Doost-Mohammady and Chowdhury 2012, Phunchongharn et al. 2010, Lee et al. 2011]. Essas técnicas permitem a utilização simultânea de diferentes canais pelas redes corporais para transmissão de dados, o que evita 
que as transmissões sofram tais interferências. Os mecanismos tradicionais e as técnicas de alocação de canais em ambientes médicos dependem de uma entidade centralizada. Essa entidade, caso seja sobrecarregada pela alta densidade das redes corporais, pode comprometer os requisitos de latência e perda de pacotes específicos para as aplicações médicas.

Esta dissertação apresentou um protocolo para camada de acesso ao meio sem fio que aloca dinamicamente canais ciente dos requisitos de aplicações médicas. $\mathrm{O}$ PDAC (Protocol for Dynamic Channel AlloCation) oferece uma solução para reduzir interferências entre redes corporais sem fio através do trabalho colaborativo de múltiplas estações base em um ambiente médico-hospitalar. O protocolo é inspirado em uma solução gulosa do problema de coloração de grafos. Desta forma, assume-se um grafo de interferências entre as estações base, no qual cada vértice representa uma estação base, cada aresta representa conflitos entre os canais utilizados pelas estações base e cada canal disponível é mapeado nas cores de um problema de coloração de grafo. De modo geral, cada estação base busca uma solução local para o problema de alocação de canais e comunica para as estações bases conflitantes o seu atual estado. Essa informação é utilizada por outras estações para auxiliar na alocação de frequências e busca reduzir interferências.

A avaliação de desempenho do protocolo foi realizada através de avaliação formal e simulações. A verificação formal ocorreu por meio da ferramenta UPPAAL. Através dela foi possível confirmar que as propriedades chave do PDAC são respeitadas e, consequentemente, assegura-se a corretude do protocolo em relação sua especificação. As simulações ocorreram através dp simulador Castalia [Boulis et al. 2011] sob cenários realistas de um ambiente médico. Os resultados mostram que o PDAC é capaz de aumentar, em média, a vazão da rede em $30 \%$ e reduzir a latência em $40 \%$, se comparado com uma abordagem de alocação de canais representativa da literatura.

Ao longo do mestrado, a relevância do PDAC foi reconhecida pela comunidade de redes de computadores através da publicação de dois artigos. O artigo "A Dynamic Channel Allocation Protocol for Medical Environment Under Multiple Base Stations" foi publicado e apresentado pelo autor dessa dissertação no IEEE Wireless Communications and Networking Conference e o "Um Protocolo de Alocação Dinâmica de Canais para Ambientes Médicos sob Múltiplas Estações Base" também publicado e apresentado pelo autor no Simpósio Brasileiro de Redes de Computadores e Sistemas Distribuídos 2017, em que esse segundo artigo foi colocado entre os dez melhores do evento, consequentemente convidado para submissão em versão estendida no "Journal of Internet Services and Applications" que se encontra em processo de revisão. Em todos artigos, foram apresentados os conceitos, revisão bibliográfica, especificação do PDAC e os resultados obtidos na avaliação de desempenho do protocolo.

\section{Ambiente Considerado}

No contexto de redes corporais, existem dois principais tipos de comunicação entre os dispositivos: a comunicação dentro da rede corporal sem fio (intra-WBAN) e a comunicação entre as redes corporais (inter-WBAN). Cada paciente possui um conjunto de sensores sem fio distribuídos ou implantados em seu corpo. Esses sensores monitoram a saúde do paciente e coletam, por exemplo, dados fisiológicos. Os sensores transmitem os dados coletados para um nó central (ex. um smartphone) situado na proximidade do paciente 
(cerca de 2 metros). Este nó central, em conjunto com os nós sensores, compõe a rede corporal sem fio, sendo a comunicação entre eles do tipo intra-WBAN. Essa comunicação dentro da WBAN ocorre por meio de transmissões de baixa potência/curto alcance (ex. Zigbee, Bluetooth). Assim, neste trabalho considera-se, sem perda de generalidade, que a comunicação intra-WBAN não causa interferências nas WBANs dos demais pacientes.

Neste trabalho, assume-se a presença de múltiplas estações base ao longo de um ambiente médico e, o paciente pode enviar dados para qualquer uma delas. O nó central, que serve como um coordenador para a rede corporal, transmite os dados recebidos para uma estação base (comunicação inter-WBAN) via Zigbee. Após a recepção dos dados, esta estação os encaminham até um repositório de dados localizado em um centro médico ou na nuvem onde são armazenados e/ou analisados. É importante lembrar que, neste trabalho, denomina-se estação base qualquer dispositivo controlador de uma rede, como exemplo, um ponto de acesso sem fio. Assim como em outros trabalhos [Doost-Mohammady and Chowdhury 2012, Phunchongharn et al. 2010], considerase um sistema no qual a estação base não possui restrições de processamento. Assume-se também que a estação base apresenta duas interfaces de rede sem fio: uma destinada à negociação de canais e outra à transmissão de dados. Essa característica permite a comunicação simultânea da estação base em dois canais sem interferência, o que aumenta a taxa de transmissão e reduz o atraso na coleta dos dados.

O nó central, por outro lado, possui apenas uma interface de comunicação sem fio. Tanto as interfaces da estação base quanto as das redes corporais podem sintonizar livremente em qualquer um dos canais disponíveis. Para uma maior economia de energia, todas as transmissões de dados ocorrem diretamente entre o nó central e a estação base, ou seja, em modo single-hop [Pešović et al. 2010].

Caso exista múltiplas estações base no ambiente, assume-se que essas podem se comunicar por meio de um canal confiável (e.g. uma conexão cabeada). Essa premissa é comum, uma vez que as estações podem se conectar por meio cabeado, com taxas de erro e latência muito inferiores às encontradas no meio sem fio. $\mathrm{O}$ foco deste trabalho é na comunicação inter-WBAN, a qual é propensa a interferências. A comunicação intra-WBAN e a comunicação cabeada entre as estações base estão fora do escopo deste trabalho.

\section{Protocolo de Alocação Dinâmica de Canais}

No PDAC, os canais de comunicação são divididos em duas categorias: canal de controle e canal de dados. As várias estações base estão interligadas no ambiente, onde cada estação possui duas interfaces de comunicação descritas anteriormente. A interface de controle está sintonizada no canal de controle conhecido por todas redes corporais, enquanto a interface de dados sintoniza de forma adaptativa dentre os canais de dados disponíveis.

Um dos diferenciais do PDAC consiste na escolha apropriada e na alocação de canais de maneira distribuída e coordenada entre as estações base. O problema de alocação de canais foi modelado como um problema de colocação de grafos, em que dois vértices adjacentes não podem ser coloridos com a mesma cor. Suponha um grafo $G=(V, E)$, os vértices no conjunto $V$ representam as estações base, e as arestas no conjunto $E$ representam conflitos entre os canais utilizados pelas estações base. Cada cor representa um canal. 
Com base em uma solução gulosa, o PDAC evita de maneira distribuída as interferências e proporciona uma divisão de carga entre as estações base.

Cada estação base mantém um controle de quais canais estão ocupados e livres com base em um histórico de alocação. Dessa maneira, a estação tem pleno controle sob o atual estado de cada canal em sua região. Conforme apresentado na Figura 1, o acesso ao meio é composto por duas fases. A primeira é chamada de fase de controle. Nela, a rede corporal negocia com a estação base um canal de dados. Ao receber a requisição da rede corporal, a estação base envia um pacote para as outras através do canal confiável existente entre as estações e informa que possui a intenção de alocar um determinado canal de dados. Caso não haja impedimentos (ex. todos os canais de dados ocupados), a estação base aloca o canal e envia um pacote para a rede corporal requisitante com tal informação, o que determina o fim da fase de controle.

A fase de transmissão ocorre após a alocação do canal. A rede corporal sintoniza no canal negociado e espera um pacote para iniciar a transmissão dos dados. Assim, a interface de dados sintoniza no canal alocado e envia o pacote esperado pela rede corporal. Recebidos os dados, a estação base envia o pacote de confirmação para a rede corporal e encerra a comunicação. A estação base anuncia para as outras estações que o canal de comunicação está livre e pode ser alocado para outras comunicações .

De modo geral, o protocolo traz uma abordagem de alocação híbrida de canais. Ele mantém uma interface da estação base em um canal estático e aloca um canal para a outra interface através de uma estratégia dinâmica. Essa abordagem tem o objetivo de iniciar uma comunicação entre a estação base e a rede corporal para evitar problemas de surdez, comuns em uma abordagem totalmente dinâmica. A partir desse contato inicial, o transmissor e receptor negociam qual canal de dados utilizar, o que evita maiores atrasos se comparado a um processo de tentativa e erro para encontrar qual canal a estação base está sintonizada [Lee et al. 2011].

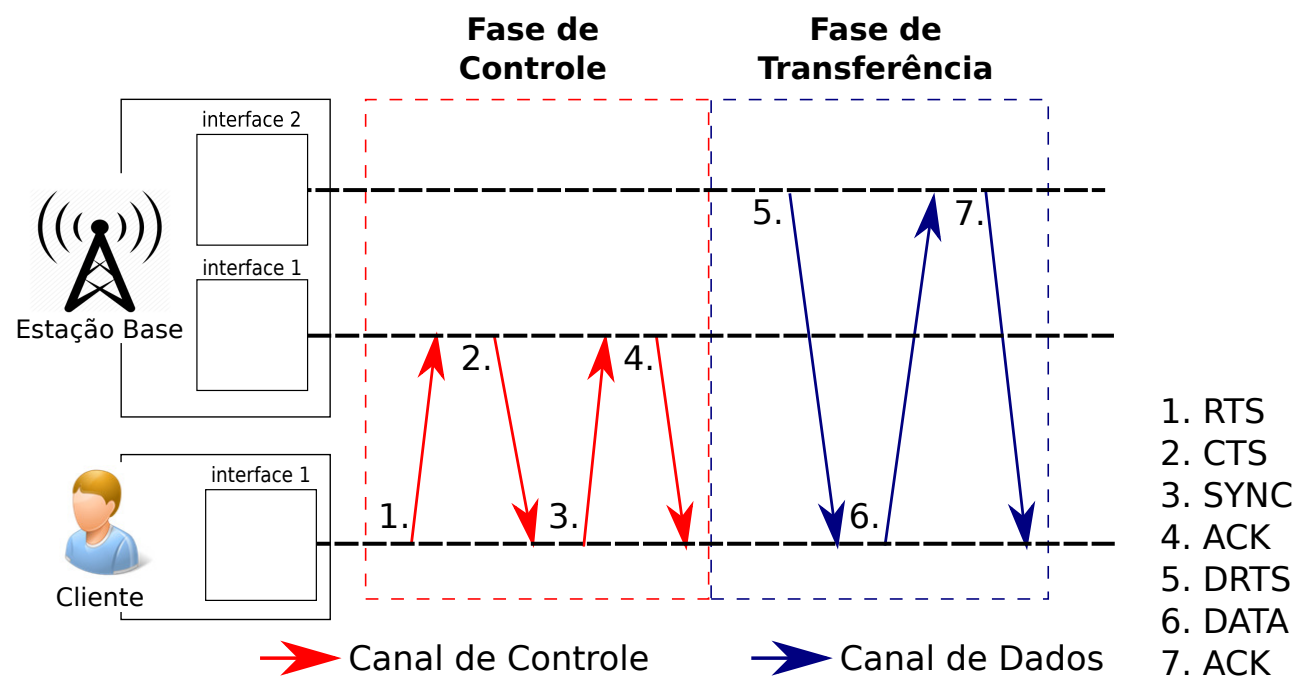

Figura 1. Diagrama de sequência do protocolo PDAC. 


\section{Avaliação do PDAC}

A avaliação do PDAC foi realizada em duas etapas. Em um primeiro momento, foi realizada uma verificação formal do protocolo. Uma verificação formal tem por finalidade garantir que as propriedades do protocolo são obedecidas. Mais ainda, verificamos ausência de estados indesejados, como deadlocks. A ferramenta utilizada para analisar formalmente o protocolo PDAC foi o Uppaal. Ela é utilizada na literatura para modelagem, simulação e verificação de sistemas através de autômatos temporizados. Todo sistema e funcionamento do PDAC foram modelados nessa ferramenta através de autômatos temporizados e através de formalismos matemáticos são utilizados para especificação e verificação do protocolo.

Dentre as propriedades do PDAC, foram verificadas: (i) Ausência de deadlock, em que através da verificação dessa propriedade, buscamos averiguar que o protocolo não entre numa situação de impasse. A outra propriedade verificada foi a (ii) Comunicação livre de interferências, em que buscamos garantir que base vizinhas não utilizem simultaneamente o mesmo canal e dois nós centrais não transmitam seus dados em um mesmo instante de tempo para uma mesma estação base através o mesmo canal de dados.

Sobre a propriedade (i), a ferramenta avaliou todos os estados alcançáveis e mostrou que o protocolo especificado não apresenta esses estados impasse, significando que, independente da situação, o protocolo é capaz de continuar sua execução. Todavia, a propriedade (ii) foi dividida em duas etapas: Inicialmente, foi realizada uma busca pela existência de alguma situação em que duas interfaces de dados não ociosas de estações base vizinhas, utilizem o mesmo canal. Como essa expressão foi verificada e não satisfeita, é demonstrado que não ocorre estações base vizinhas que operem no mesmo canal simultaneamente. Em um segundo momento, foi realizada uma busca por uma situação em que nós centrais não ociosos se comunicam simultaneamente com a mesma estação base e utilizem o mesmo canal. Novamente essa expressão foi verificada e não satisfeita, o que implica em um modelo sem a ocorrência de clientes que se comunicam com a estação base através do mesmo canal de dados. Logo, uma vez recebida a autorização de acesso a um canal de dados, seu acesso é exclusivo para um determinado nó central.

Após a verificação da corretude do protocolo, a segunda etapa de avaliação do PDAC ocorreu através de simulações de cenários médicos-hospitalar através do Castalia [Boulis et al. 2011]. O Castalia é um simulador de redes baseado no OMNeT++ [Varga et al. 2001] e é utilizado por pesquisadores para testes de algoritmos e protocolos com base em modelos reais de canal sem fio e rádio. As simulações são conduzidas em dois ambientes médicos realistas: um departamento de emergência sobrecarregado e um ambiente calmo, como uma sala de radiologia. Ambos os cenários contam com uma área de $30 \mathrm{~m}^{2}$ e estações base fixas de forma que não ocorra problemas de terminal oculto. Os pacientes e seus nós centrais são distribuídos no ambiente conforme uma distribuição uniforme e estão no raio de alcance das estações base.

O objetivo das simulações são: verificar a eficiência do PDAC em relação a outros protocolos e em um segundo momento, observar o comportamento do PDAC em um ambiente com múltiplas estações base, sendo esta segunda, a principal característica do PDAC. Sendo assim, comparamos o protocolo PDAC com o protocolo DCAA [Lee et al. 2011], um dos mais representativos da literatura quando se refere à alocação dinâmica de canais e em um segundo momento, nós avaliamos o impacto do 


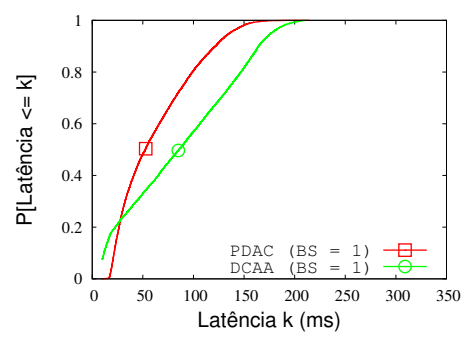

(a) Radiologia

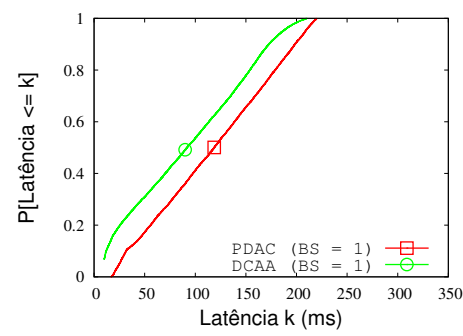

(b) Dpt. Emergência

Figura 2. Distribuição de latência apresentada pelos protocolos DCAA e PDAC.

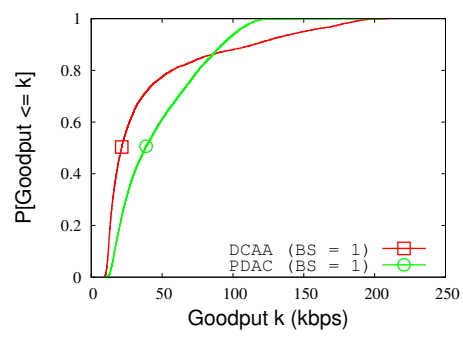

(a) Radiologia

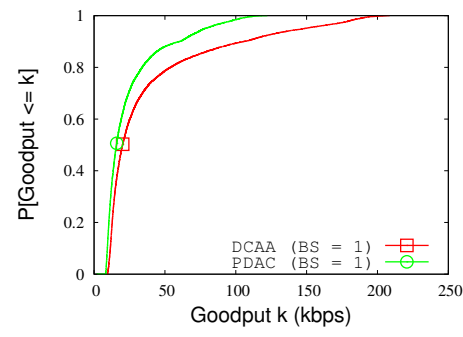

(b) Dpt. Emergência

Figura 3. Distribuição de goodput apresentada pelos protocolos DCAA e PDAC.

número de estações base disponíveis no desempenho do novo protocolo.

De forma a realizar uma comparação justa, para a primeira simulação limitamos o PDAC a uma configuração equivalente à suportada pelo DCAA. Neste caso, o PDAC não faz uso de múltiplas estações e ambos os protocolos estão limitados ao uso de quatro canais de dados. Alteramos o número de canais disponíveis e as conclusões apresentadas se mantém. Omitimos tais resultados por restrições de espaço.

A Figura 3 apresenta a função de distribuição de probabilidade acumulada da latência em dois cenários distintos, um departamento de emergência e uma sala de radiologia. Observe que, para um cenário de carga de dados baixa (sala de radiologia), o protocolo PDAC apresenta desempenho melhor que o protocolo DCAA. Neste caso, o novo protocolo tem uma latência média 1,4 vezes melhor que o protocolo DCAA. O protocolo PDAC apresentou uma taxa de entrega de 99,9\% dos pacotes, enquanto o protocolo DCAA apresentou menos de $88 \%$ de taxa de entrega. Mais ainda, em mais de $45 \%$, o goodput apresentado pelo PDAC foi de pelo menos $50 \mathrm{kbps}$, enquanto que em menos de $25 \%$ dos casos o DCAA atinge esta marca.

Em um cenário de alta carga de dados, como em um departamento de emergência médica, há uma degradação do desempenho em ambos os protocolos. Mais ainda, os pacotes entregues pelo DCAA têm latência média menor que a do novo protocolo. Porém, a taxa de perda que o PDAC apresenta é menor que o DCAA. Enquanto este apresentou cerca de $30 \%$ de perda, o PDAC teve em torno de $24 \%$. Os valores de goodput encontrados seguem a mesma tendência da latência. Cerca de $25 \%$ dos casos no DCAA apresentam mais de $50 \mathrm{kbps}$, enquanto cerca de $18 \%$ do PDAC atingem a mesma marca. 
A principal característica do PDAC é o uso de múltiplas estações base, o que distribui a carga entre elas. Assim, nós avaliamos seu comportamento através da variação do número de estações base até 4, onde o grafo de interferências entre as estações base é completo, e o número de canais disponíveis para comunicação até 16. Nesta análise, o cenário de radiologia foi desconsiderado uma vez que uma única estação base foi capaz de atender seus requisitos. As Figuras 4 e 5 apresentam a função de distribuição de probabilidade acumulada da latência e do goodput, respectivamente. De forma geral, quanto maior o número de estações base utilizada, melhor é o desempenho do novo protocolo. Da mesma forma, o aumento no número de canais disponíveis para comunicação gera ganhos consideráveis. Por exemplo, a partir de duas estações base, com 8 canais disponíveis para transmissão de dados, o PDAC foi capaz de atender aos requisitos das aplicações médicas, apresentou uma taxa de entrega de pacotes de $99.9 \%$. Neste caso, a latência média foi de $86,45 \mathrm{~ms}$ e o goodput foi de $33,36 \mathrm{kbps}$.

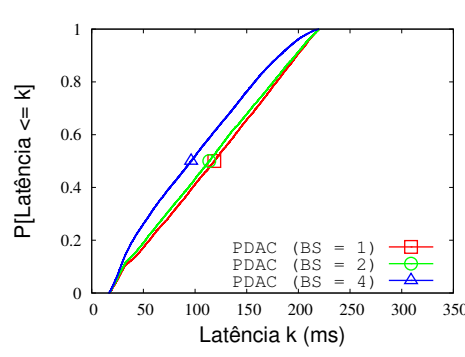

(a) 4 Canais

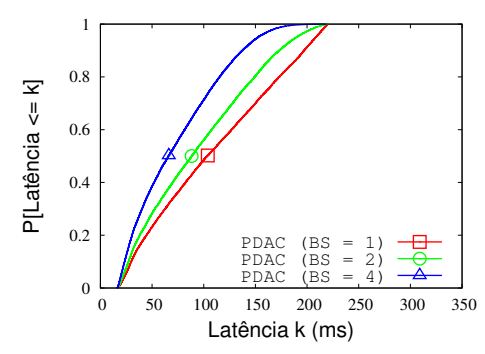

(b) 8 Canais

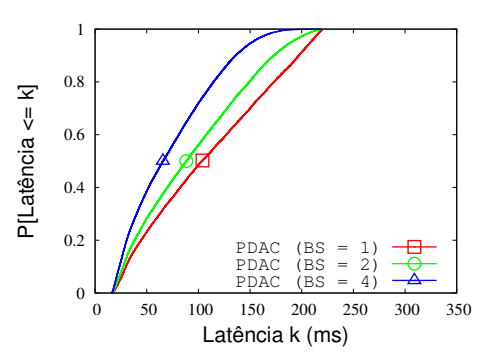

(c) 16 Canais

Figura 4. Latência em relação ao número de canais disponíveis.

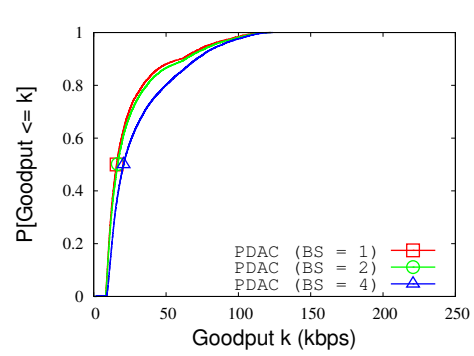

(a) 4 Canais

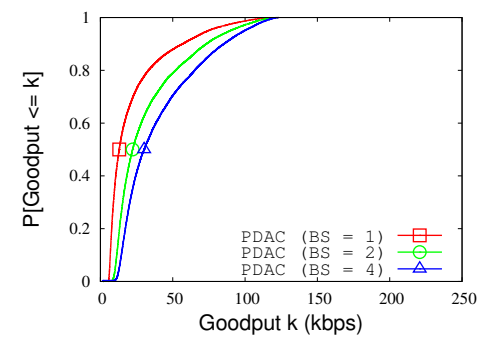

(b) 8 Canais

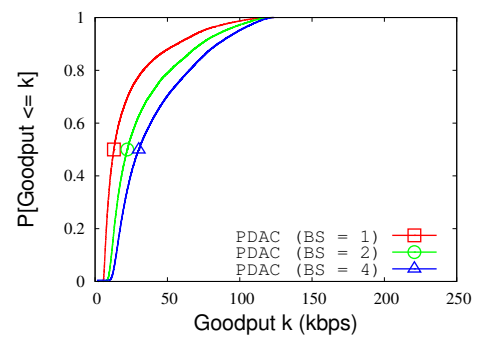

(c) 16 Canais

Figura 5. Goodput em relação ao número de canais disponíveis.

Apesar do ganho que ambos os fatores trazem, notamos que o crescimento no número de canais tem menor impacto no desempenho que o número de estações. Ao se aumentar o número de estações, esperava-se que ocorresse uma divisão de carga entre elas. Por outro lado, mesmo com a existência de múltiplos canais, a fase inicial do protocolo não é paralelizável, o que limita os ganhos dentro de uma mesma estação.

\section{Conclusões}

Nesta dissertação de mestrado, foi proposto o protocolo PDAC para a camada de acesso ao meio. Esse protocolo possui como objetivo oferecer uma solução para reduzir o problema de interferências relacionado ao ambiente médico. Foi evidenciado que, quando não há uma coordenação entre as redes corporais, elas são capazes de utilizar simultaneamente 
o mesmo canal para uma transferência de dados para uma mesma estação base, o que ocasiona em interferências que geram uma queda do desempenho na rede e provocam um aumento da latência e perda de pacotes.

O protocolo PDAC, diferente de abordagens tradicionais, trabalha de maneira distribuída. Com suporte a múltiplas estações base, ele fornece canais de maneira dinâmica e exclusiva para as redes corporais de forma a evitar transferências simultâneas através de uma mesmo canal. Devido a coordenação entre as estações base, as transferências ocorrem de maneira eficiente e com uma sobrecarga muito mais baixa para cada estação base, o que impacta positivamente no desempenho da rede.

O protocolo foi avaliado de duas maneiras diferentes: foi realizado uma verificação formal com base em autômatos temporizados e simulações utilizando o simulador Castalia. A avaliação formal evidenciou a corretude do PDAC, demonstrando que o mesmo não possui deadlocks e tem suas propriedades satisfeitas. As simulações por sua vez, demonstraram melhorias substanciais no o desempenho da rede. Mostrou-se que sob a presença de uma única estação base, o PDAC foi capaz de, em média, aumentar a vazão em $30 \%$ e reduzir a latência em $40 \%$, quando comparado com os protocolos do estado-da-arte. Assim, pode-se concluir da dissertação que o uso de múltiplas estações base para o controle do uso de canais no ambiente médico juntamente com um processo de alocação dinâmico e cooperativo de canais é capaz de aumentar substancialmente o desempenho das redes corporais, uma vez que a exclusividade do acesso a um canal de transmissão evita interferências que degradam a latência e a taxa de entrega.

\section{Referências}

Baker, S. D. and Hoglund, D. H. (2008). Medical-grade, mission-critical wireless networks [designing an enterprise mobility solution in the healthcare environment. IEEE Eng Med Biol., 27(2):86-95.

Boulis, A. et al. (2011). Castalia: A simulator for wireless sensor networks and body area networks. NICTA: National ICT Australia.

Doost-Mohammady, R. and Chowdhury, K. R. (2012). Transforming healthcare and medical telemetry through cognitive radio networks. IEEE Wirel Commun., 19(4):67-73.

Fang, G., Dutkiewicz, E., Yu, K., Vesilo, R., and Yu, Y. (2010). Distributed inter-network interference coordination for wireless body area networks. In IEEE GLOBECOM, pages $1-5$.

Lee, B., Yun, J., and Han, K. (2011). Dynamic channel adjustable asynchronous cognitive radio mac protocol for wireless medical body area sensor networks. In Communication and Networking, volume 266, pages 338-345. Springer.

Pešović, U. M., Mohorko, J. J., Benkič, K., and Čučej, Ž. F. (2010). Single-hop vs. multihop-energy efficiency analysis in wireless sensor networks. In Telecommun. Forum.

Phunchongharn, P., Hossain, E., Niyato, D., and Camorlinga, S. (2010). A cognitive radio system for e-health applications in a hospital environment. IEEE Wirel Commun., 17(1):20-28.

Varga, A. et al. (2001). The omnet++ discrete event simulation system. In Proceedings of the European simulation multiconference (ESM'2001), volume 9, page 65. sn.

Yu, J.-Y., Liao, W.-C., and Lee, C.-Y. (2006). A MT-CDMA based wireless body area network for ubiquitous healthcare monitoring. In IEEE Biomedical Circuits and Systems Conference, pages 98-101. IEEE. 